und finanziellen Hemmnisse für die Errichtung der nötigen Anlagen zu überwinden.

Die grossen Schwierigkeiten bei der Realisation neuer Anlagen und insbesondere die langwierigen Verfahren, welche sich ergeben, wenn für Deponien oder Behandlungsanlagen zuerst Umzonungen nötig sind, bestätigen die Notwendigkeit, die Abfallentsorgung in Zukunft längerfristig zu planen. Nur wenn die Standorte von Deponien und Verbrennungsanlagen innerhalb kantonaler Planungen langfristig gesichert werden, können solche Anla- gen ohne zeitraubende Umzonungsverfahren auch erstellt werden.

\section{Ausblick}

Der Übergang zu einer ökologischen Abfallwirtschaft ist, wie diese Übersicht zeigt, nicht so sehr die Frage einer einzelnen Massnahme. Die vielen aktuellen Probleme lassen sich nicht durch Schlagworte lösen; hingegen gilt es, einzelne Produk- tionsverfahren zu optimieren, technische Lösungen zum Verwerten und Behandeln von Abfällen zu erarbeiten, finanzielle und organisatorische Voraussetzungen für eine zuverlässige Entsorgung zu schaffen.

Längerfristig resultiert daraus eine leistungsfähige Infrastruktur, welche schliesslich nicht nur eine umweltgerechte Entsorgung im Inland erlaubt, sondern selbst auch Signale abgibt, mit denen sich die zukünftige Produktion von vorneherein entsorgungs- und umweltgerecht steuern lässt.

\title{
Abfallszene Region Basel
}

\section{Kurzfassung}

\section{Heinz Peter*}

In der Kehrichtverbrennungsanlage $\mathrm{Ba}-$ sel (KVA) werden jährlich 200000 t Siedlungsabfälle verbrannt. Davon stammen aber nur $120000 \mathrm{t}$ aus dem Kanton BaselStadt selber. $50000 \mathrm{t}$ stammen aus dem Kanton Basel-Landschaft, $20000 \mathrm{t}$ aus dem benachbarten Elsass (das seinerseits jährlich etwa $60000 \mathrm{t}$ KVA-Schlacke in eine an der Schweizer Grenze gelegene Deponie übernimmt), der Rest stammt aus andern zur KVA BS orientierten Gebieten der Region. In der vom Kanton BaselLandschaft erstellten und betriebenen $\mathrm{Re}$ aktordeponie Elbisgraben bei Liestal wer-

\footnotetext{
* Korrespondenz: H. Peter

Amt für Umweltschutz und Energie

Kanton Basel-Landschaft

CH-4410 Liestal
}

den anderseits jährlich rund $130000 \mathrm{t} \mathrm{Ab-}$ fälle abgelagert. Davon stammen rund $42 \%$ aus Haushaltungen, $28 \%$ aus Industrie und Gewerbe, $13 \%$ sind sog. Shredderabfälle aus Altautoverwertungsanlagen, rund $7 \%$ sind Klärschlamm und $10 \%$ Schlacken und Aschen.

Im Referat wird aufgezeigt, wie sich die verantwortlichen Kantonsregierungen BS/ BL die Zukunft vorstellen, wie die Mengenperspektiven aussehen und wie die klassischen Strategien Vermeiden, Wiederverwerten, Entsorgen praktisch angepackt werden sollen. Insbesondere gilt es, den sich abzeichnenden Notstand der mangelnden Verbrennungskapazitäten möglichst rasch, effektiv und umweltschonend zu beheben. Die regionale Zusammenarbeit, die schon bis anhin gut spielte, wird in
Chimia 44 (1990) 173-175

(C) Schweiz. Chemiker-Verband; ISSN 0009-4293

\section{Rückgewinnung von CKW aus Destillationsrückständen}

René Meier*

\section{Einleitung}

Als Benützer von Anlagen, bei denen Chlorkohlenwasserstoffe od. sog. CKW's eingesetzt werden, sind wir es unseren Mit-

\footnotetext{
* Korrespondenz: R. Meier

MENTEC AG

Reckenbühlstr. 21, CH-6005 Luzern
}

menschen, besonders aber unsern Nachfahren gegenüber schuldig, deren Emissionen so gering wie möglich zu halten. Bei der Suche nach geeigneten Methoden zur Reduktion von CKW-Emissionen müssen sämtliche damit verbundenen Emissionen, dazu gehört auch der Energie- verbrauch, in unsere Überlegungen miteinbezogen werden.

\footnotetext{
Allgemeines

Nachfolgend wird ein neues, wegweisendes Verfahren für die Verfestigung von CKW-haltigen Destillationsrückständen vorgestellt, bei dem die schädlichen CKW's nahezu restlos zurückgewonnen werden. Die Wirtschaftlichkeit dieses Verfahrens soll durch ein Beispiel aus der Leiterplattenfertigung aufgezeigt werden. Gerade bei der Leiterplattenfertigung fallen grosse Mengen von verschmutzten CKW's an. Diese werden in Destillatoren gereinigt und danach dem Prozess direkt wieder zugeführt (Fig. I).
} 
und finanziellen Hemmnisse für die Errichtung der nötigen Anlagen zu überwinden.

Die grossen Schwierigkeiten bei der Realisation neuer Anlagen und insbesondere die langwierigen Verfahren, welche sich ergeben, wenn für Deponien oder Behandlungsanlagen zuerst Umzonungen nötig sind, bestätigen die Notwendigkeit, die Abfallentsorgung in Zukunft längerfristig zu planen. Nur wenn die Standorte von Deponien und Verbrennungsanlagen innerhalb kantonaler Planungen langfristig gesichert werden, können solche Anla- gen ohne zeitraubende Umzonungsverfahren auch erstellt werden.

\section{Ausblick}

Der Übergang zu einer ökologischen Abfallwirtschaft ist, wie diese Übersicht zeigt, nicht so sehr die Frage einer einzelnen Massnahme. Die vielen aktuellen Probleme lassen sich nicht durch Schlagworte lösen; hingegen gilt es, einzelne Produk- tionsverfahren zu optimieren, technische Lösungen zum Verwerten und Behandeln von Abfällen zu erarbeiten, finanzielle und organisatorische Voraussetzungen für eine zuverlässige Entsorgung zu schaffen.

Längerfristig resultiert daraus eine leistungsfähige Infrastruktur, welche schliesslich nicht nur eine umweltgerechte Entsorgung im Inland erlaubt, sondern selbst auch Signale abgibt, mit denen sich die zukünftige Produktion von vorneherein entsorgungs- und umweltgerecht steuern lässt.

\section{Abfallszene Region Basel}

\section{Kurzfassung}

\section{Heinz Peter*}

In der Kehrichtverbrennungsanlage $\mathrm{Ba}-$ sel (KVA) werden jährlich 200000 t Siedlungsabfälle verbrannt. Davon stammen aber nur $120000 \mathrm{t}$ aus dem Kanton BaselStadt selber. $50000 \mathrm{t}$ stammen aus dem Kanton Basel-Landschaft, $20000 \mathrm{t}$ aus dem benachbarten Elsass (das seinerseits jährlich etwa $60000 \mathrm{t}$ KVA-Schlacke in eine an der Schweizer Grenze gelegene Deponie übernimmt), der Rest stammt aus andern zur KVA BS orientierten Gebieten der Region. In der vom Kanton BaselLandschaft erstellten und betriebenen $\mathrm{Re}$ aktordeponie Elbisgraben bei Liestal wer-

\footnotetext{
* Korrespondenz: H. Peter

Amt für Umweltschutz und Energie

Kanton Basel-Landschaft

CH-4410 Liestal
}

den anderseits jährlich rund $130000 \mathrm{t} \mathrm{Ab-}$ fälle abgelagert. Davon stammen rund $42 \%$ aus Haushaltungen, $28 \%$ aus Industrie und Gewerbe, $13 \%$ sind sog. Shredderabfälle aus Altautoverwertungsanlagen, rund $7 \%$ sind Klärschlamm und $10 \%$ Schlacken und Aschen.

Im Referat wird aufgezeigt, wie sich die verantwortlichen Kantonsregierungen BS/ BL die Zukunft vorstellen, wie die Mengenperspektiven aussehen und wie die klassischen Strategien Vermeiden, Wiederverwerten, Entsorgen praktisch angepackt werden sollen. Insbesondere gilt es, den sich abzeichnenden Notstand der mangelnden Verbrennungskapazitäten möglichst rasch, effektiv und umweltschonend zu beheben. Die regionale Zusammenarbeit, die schon bis anhin gut spielte, wird in
Chimia 44 (1990) 173-175

(C) Schweiz. Chemiker-Verband; ISSN 0009-4293

\section{Rückgewinnung von CKW aus Destillationsrückständen}

René Meier*

\section{Einleitung}

Als Benützer von Anlagen, bei denen Chlorkohlenwasserstoffe od. sog. CKW's eingesetzt werden, sind wir es unseren Mit-

\footnotetext{
* Korrespondenz: R. Meier

MENTEC AG

Reckenbühlstr. 21, CH-6005 Luzern
}

menschen, besonders aber unsern Nachfahren gegenüber schuldig, deren Emissionen so gering wie möglich zu halten. Bei der Suche nach geeigneten Methoden zur Reduktion von CKW-Emissionen müssen sämtliche damit verbundenen Emissionen, dazu gehört auch der Energie- verbrauch, in unsere Überlegungen miteinbezogen werden.

\footnotetext{
Allgemeines

Nachfolgend wird ein neues, wegweisendes Verfahren für die Verfestigung von CKW-haltigen Destillationsrückständen vorgestellt, bei dem die schädlichen CKW's nahezu restlos zurückgewonnen werden. Die Wirtschaftlichkeit dieses Verfahrens soll durch ein Beispiel aus der Leiterplattenfertigung aufgezeigt werden. Gerade bei der Leiterplattenfertigung fallen grosse Mengen von verschmutzten CKW's an. Diese werden in Destillatoren gereinigt und danach dem Prozess direkt wieder zugeführt (Fig. I).
} 
und finanziellen Hemmnisse für die Errichtung der nötigen Anlagen zu überwinden.

Die grossen Schwierigkeiten bei der Realisation neuer Anlagen und insbesondere die langwierigen Verfahren, welche sich ergeben, wenn für Deponien oder Behandlungsanlagen zuerst Umzonungen nötig sind, bestätigen die Notwendigkeit, die Abfallentsorgung in Zukunft längerfristig zu planen. Nur wenn die Standorte von Deponien und Verbrennungsanlagen innerhalb kantonaler Planungen langfristig gesichert werden, können solche Anla- gen ohne zeitraubende Umzonungsverfahren auch erstellt werden.

\section{Ausblick}

Der Übergang zu einer ökologischen Abfallwirtschaft ist, wie diese Übersicht zeigt, nicht so sehr die Frage einer einzelnen Massnahme. Die vielen aktuellen Probleme lassen sich nicht durch Schlagworte lösen; hingegen gilt es, einzelne Produk- tionsverfahren zu optimieren, technische Lösungen zum Verwerten und Behandeln von Abfällen zu erarbeiten, finanzielle und organisatorische Voraussetzungen für eine zuverlässige Entsorgung zu schaffen.

Längerfristig resultiert daraus eine leistungsfähige Infrastruktur, welche schliesslich nicht nur eine umweltgerechte Entsorgung im Inland erlaubt, sondern selbst auch Signale abgibt, mit denen sich die zukünftige Produktion von vorneherein entsorgungs- und umweltgerecht steuern lässt.

\section{Abfallszene Region Basel}

\section{Kurzfassung}

\section{Heinz Peter*}

In der Kehrichtverbrennungsanlage $\mathrm{Ba}-$ sel (KVA) werden jährlich 200000 t Siedlungsabfälle verbrannt. Davon stammen aber nur $120000 \mathrm{t}$ aus dem Kanton BaselStadt selber. $50000 \mathrm{t}$ stammen aus dem Kanton Basel-Landschaft, $20000 \mathrm{t}$ aus dem benachbarten Elsass (das seinerseits jährlich etwa $60000 \mathrm{t}$ KVA-Schlacke in eine an der Schweizer Grenze gelegene Deponie übernimmt), der Rest stammt aus andern zur KVA BS orientierten Gebieten der Region. In der vom Kanton BaselLandschaft erstellten und betriebenen $\mathrm{Re}$ aktordeponie Elbisgraben bei Liestal wer-

\footnotetext{
* Korrespondenz: H. Peter

Amt für Umweltschutz und Energie

Kanton Basel-Landschaft

CH-4410 Liestal
}

den anderseits jährlich rund $130000 \mathrm{t} \mathrm{Ab-}$ fälle abgelagert. Davon stammen rund $42 \%$ aus Haushaltungen, $28 \%$ aus Industrie und Gewerbe, $13 \%$ sind sog. Shredderabfälle aus Altautoverwertungsanlagen, rund $7 \%$ sind Klärschlamm und $10 \%$ Schlacken und Aschen.

Im Referat wird aufgezeigt, wie sich die verantwortlichen Kantonsregierungen BS/ BL die Zukunft vorstellen, wie die Mengenperspektiven aussehen und wie die klassischen Strategien Vermeiden, Wiederverwerten, Entsorgen praktisch angepackt werden sollen. Insbesondere gilt es, den sich abzeichnenden Notstand der mangelnden Verbrennungskapazitäten möglichst rasch, effektiv und umweltschonend zu beheben. Die regionale Zusammenarbeit, die schon bis anhin gut spielte, wird in
Chimia 44 (1990) 173-175

(C) Schweiz. Chemiker-Verband; ISSN 0009-4293

\section{Rückgewinnung von CKW aus Destillationsrückständen}

René Meier*

\section{Einleitung}

Als Benützer von Anlagen, bei denen Chlorkohlenwasserstoffe od. sog. CKW's eingesetzt werden, sind wir es unseren Mit-

\footnotetext{
* Korrespondenz: R. Meier

MENTEC AG

Reckenbühlstr. 21, CH-6005 Luzern
}

menschen, besonders aber unsern Nachfahren gegenüber schuldig, deren Emissionen so gering wie möglich zu halten. Bei der Suche nach geeigneten Methoden zur Reduktion von CKW-Emissionen müssen sämtliche damit verbundenen Emissionen, dazu gehört auch der Energie- verbrauch, in unsere Überlegungen miteinbezogen werden.

\footnotetext{
Allgemeines

Nachfolgend wird ein neues, wegweisendes Verfahren für die Verfestigung von CKW-haltigen Destillationsrückständen vorgestellt, bei dem die schädlichen CKW's nahezu restlos zurückgewonnen werden. Die Wirtschaftlichkeit dieses Verfahrens soll durch ein Beispiel aus der Leiterplattenfertigung aufgezeigt werden. Gerade bei der Leiterplattenfertigung fallen grosse Mengen von verschmutzten CKW's an. Diese werden in Destillatoren gereinigt und danach dem Prozess direkt wieder zugeführt (Fig. I).
} 


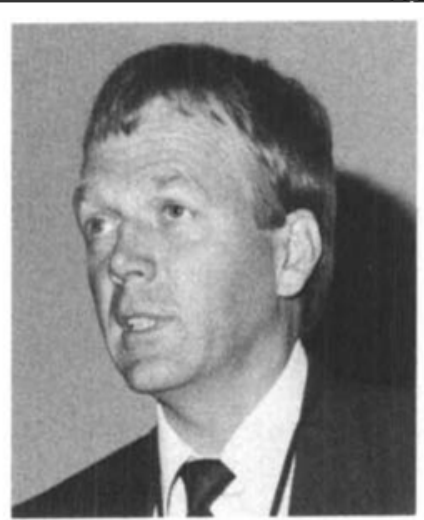

René Meier : Ingenieur, ist 1942 in Basel geboren, wo er aufgewachsen ist und die Schulen durchlaufen hat. Er ist Leiter der Firma MENTEC AG, die sich mit Umwelt- und Verfahrenstechnik befasst und ihren Sitz in Luzern hat. Seit 1980 beschäftigt sich die Firma MENTEC $A G$ mit der Entwicklung und dem Bau von Anlagen zu einer wirtschaftlichen Rückgewinnung von Lösungsmitteln.

Fig. I zeigt die Stoffbilanz eines Destillators: A Zulauf verschmutztes Lösungsmittel; B sauberes Lösungsmittel - zurück zum Prozess; C Destillationsrückstand. Die Aufarbeitung dieser Rückstände ist Gegenstand dieses Vortrages. Doch vorerst kurz einige Bemerkungen zur Destillation.

Aus Fig. 2 ist zu sehen, dass die Destillationsrückstände noch beträchtliche Mengen an CKW's enthalten. Ferner erkennt man, dass mit der Wahl eines geeigneten Destillators der Lösungsmittelverlust stark reduziert werden kann.

Bei der konventionellen Destillation besteht der Rückstand aus nur $10 \%$ FS und $90 \%$ Lösungsmittel und bei der Vakuum-

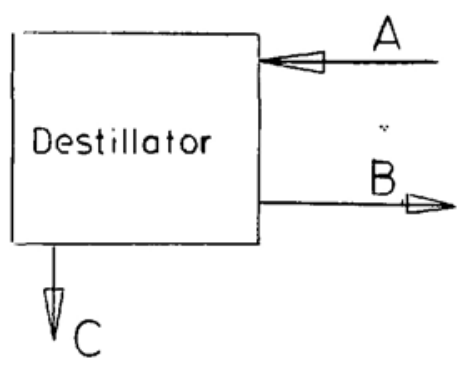

Fig. 1. Stoffbilanz eines Destillators
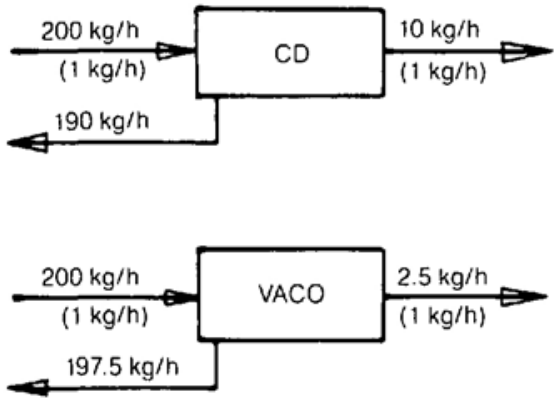

Fig. 2. Mengenflussvergleich von 2 Destillations-Systemen. $\mathrm{CD}=$ konventionelle Destillation, $\mathrm{VACO}=$ Vakuumdestillation. destillation immerhin aus $40 \%$ FS und $60 \%$ Lösungsmittel. Beim Einsatz eines Vakuumdestillators anstelle eines konventionellen Destillators würde bei einer Betriebszeit von z.B. $6000 \mathrm{Bh} / \mathrm{Jahr}$ der Destillationsrückstand von $60000 \mathrm{~kg}$ auf 15000 $\mathrm{kg} / \mathrm{Jahr}$ reduziert. Der Lösungsmittelverlust ist jedoch auch bei einem Vakuumdestillator immer noch beträchtlich. Die $15000 \mathrm{~kg}$ Rückstand aus dem Vakuumdestillator enthalten immerhin noch $c a .9000$ $\mathrm{kg}$ Lösungsmittel.

Fig. 3 zeigt einen Vakuumdestillator Typ VACUMEX. Ein solcher Vakuumdestillator, wie er in der Leiterplattenfertigung oft eingesetzt wird, produziert pro Jahr ca. 30 Fass Rückstand, vergleichsweise produziert ein konventioneller Déstillator ca. 200 Fass/Jahr. Die Destillationsrückstände aus der Leiterplattenfertigung bestehen aus Photoresist und CKW's (Methylenchlorid und 1,1,1-Trichloräthan). Bei einem Feststoffgehalt von $c a$. $50 \%$ sind diese Destillationsrückstände nicht mehr fliessfähig, so dass zur Rückgewinnung der CKW's spezielle Massnahmen getroffen werden müssen.

Konventionelle Systeme eignen sich nicht für eine vollständige Rückgewinnung von CKW's aus Photoresist. Sobald dem Rückstand das Lösungsmittel entzogen wird, erhärtet dieser. Der erhärtete Photoresist wirkt isolierend und erschwert das Eindringen der erforderlichen Wärme, welche zur Verdampfung des Lösungsmittels notwendig ist. UUberhöhte Heiztemperaturen führen zu Verbrennungen des Photoresists und zu Zersetzungen des Lösungsmittels sowie zur Bildung von Chloriden. Deshalb ist es nicht sinnvoll, auf diese Weise die CKW's aus dem Destillationsrückstand zurückzugewinnen.

Fig. 4 zeigt uns ein SOLIDEX-Gerät schematisch dargestellt. Es bedeuten:

1 Verdampfer

2 Behälter für Destillationsrückstand

3 Abfallbeutel

4 Kondensator

5 Kondensatauffangbehälter

6 Vakuumpumpe

7 Mikrowellengenerator

8 Schlammpumpe

A Destillationsrückstand

B Destillat (sauberes Lösungsmittel)

$\mathrm{C}$ trockener Rückstand

Mit dem SOLIDEX steht ein neues, patentiertes Verfahren zur Verfügung, welches die obgenannten Probleme auf elegante Art und Weise löst. Beim Solidex wird ein Verfahren verwendet, bei welchem das Lö-

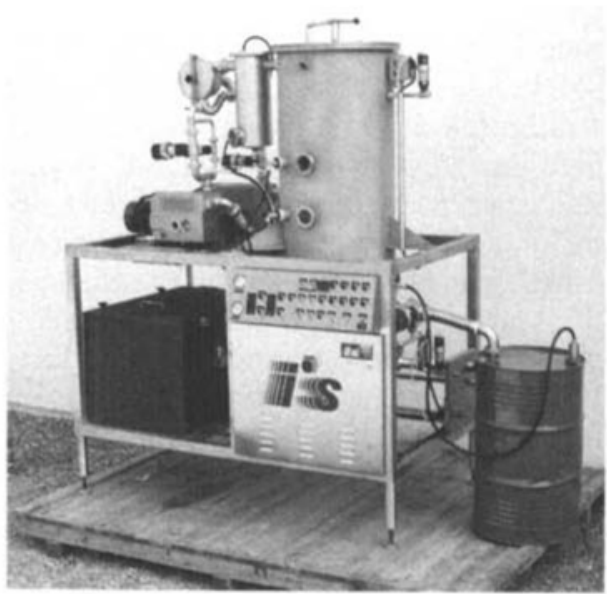

Fig. 3. VACUMEX

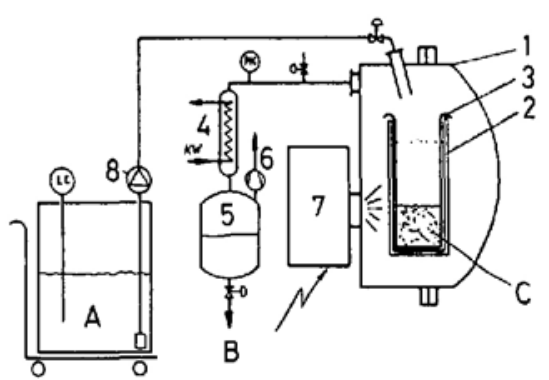

Fig. 4. Schematische Darstellung des SOLIDEX

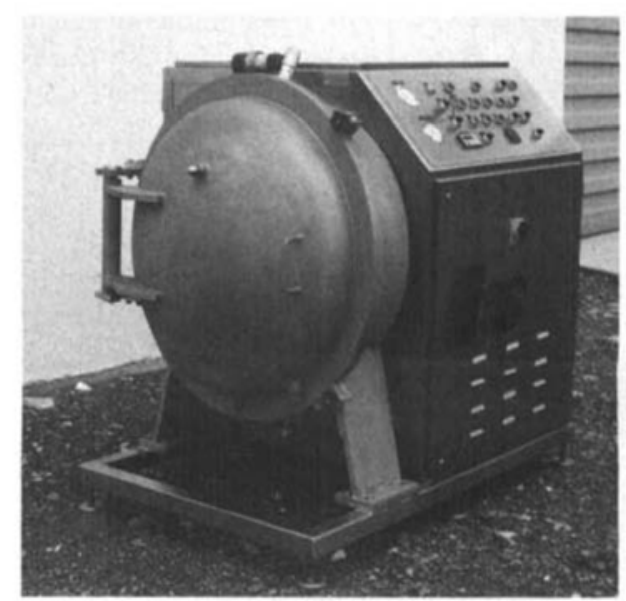

Fig. 5. SOLIDEX (geschlossen)

sungsmittel unter Vakuum abgedampft und kondensiert wird. Die dazu benötigte Wärmeenergie wird über Mikrowellengeneratoren zugeführt. Die Mikrowellen werden vom Photoresist nicht absorbiert und können dadurch direkt zur gleichmässigen Erwärmung des Lösungsmittels im Rückstand genutzt werden. Ferner kann die Mikrowellenenergie zu fast $100 \%$ für die Verdampfung des Lösungsmittels genützt werden. Dadurch verdampft das Lö-
Tabelle. Leistungsbeispiel für ein SOLIDEX-Gerät

\section{Tagesleistung}

Zurückgewonnenes Lösungsmittel

Trockener Rückstand

Lösungsmittelrückgewinnung bei 250 Arbeitstagen/Jahr Reduktion des Abfalls

Reduktion des Sonderabfalls

Entsorgungskosten für Destillationsrückstände mit mehr als 2\% Chlor-Gehalt

Energiekosten SOLIDEX

el. Energie

$$
\begin{array}{r}
3 \text { Chargen à } 50 \mathrm{~kg}=150 \mathrm{~kg} \\
3 \times 35 \mathrm{~kg}=105 \mathrm{~kg} \\
3 \times 15 \mathrm{~kg}=45 \mathrm{~kg} \\
250 \times 105 \mathrm{~kg}=26250 \mathrm{~kg} / \mathrm{J} \\
=26250 \mathrm{~kg} / \mathrm{J} \\
250 \times 150 \mathrm{~kg}=37500 \mathrm{~kg} / \mathrm{J} \\
\text { ca. Fr. } 1800 / \mathrm{t}
\end{array}
$$

ca. $6 \mathrm{kWh}$ pro Charge $=0,17 \mathrm{kWh} / \mathrm{kg}$ zurückgew. Lösungsmittel 
sungsmittel restlos. Das geschlossene System erlaubt eine vollständige Rückgewinnung des Lösungsmittels. Als Resultat erhält man einen trockenen, porösen, lösungsmittelfreien Block aus Photoresist.

\section{Funktionsbeschreibung}

Der Destillationsrückstand wird durch eine Schlammpumpe (8) in den Behälter (2), der mit einem Abfallbeutel (3) ausgekleidet ist, gepumpt. Das Fassungsvermögen dieses Behälters beträgt $c a$. 501 . Die Vakuumpumpe (6) setzt das gesamte System unter Vakuum. Der Mikrowellengenerator (7) liefert die erforderliche Energie, um das Lösungsmittel auszudampfen. Das ausgedampfte Lösungsmittel wird im Kondensator (4) kondensiert und in einem Auffangbehälter (5) aufgefangen. Am Ende des Zyklusses schaltet das Gerät automatisch aus. Der erhärtete, trockene, lösungsmittelfreie Rückstand kann auf einfache Weise mit dem Abfallbeutel aus dem SOLIDEX entnommen werden.

Das SOLIDEX-Gerät (Fig. 5 und 6) ist selbstverständlich mit allen erforderlichen Sicherheitsvorrichtungen ausgerüstet, wie z.B.

- einem Infrarotsensor, welcher die Temperatur des Rückstandes im SOLIDEX erfasst und den Mikrowellengenerator ausschaltet, wenn eine vorgegebene Solltemperatur erreicht ist.

- Der Mikrowellengenerator kann nur senden, wenn die Türe geschlossen und das vorgegebene Vakuum vorhanden ist.

Die SOLIDEX-Geräte sind vollautomatisch - ausser der Entnahme des Kuchens am Ende des Zyklusses und dem Einlegen

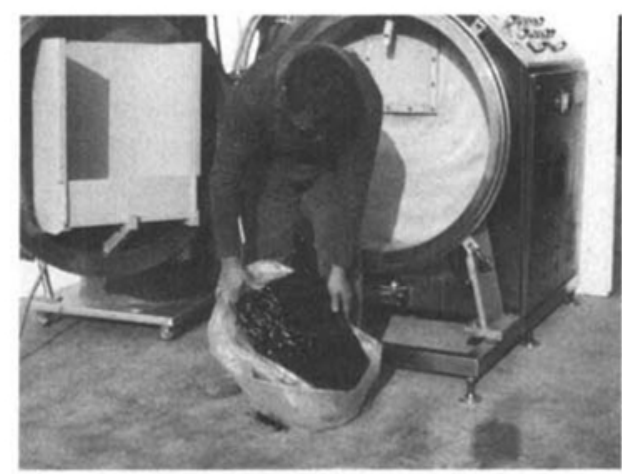

Fig. 6. SOLIDEX (offen)

eines neuen Abfallbeutels. Die produktberührten Teile sind aus Inox oder PP. Man sieht in der Tür den Aufnahmebehälter aus PP. Es ist zu sehen, dass der trockene Rückstand (Fig. 7) auf einfache Weise mit dem Abfallbeutel aus dem Gerät entnommen werden kann. Die gleichmässige, durchgehende, poröse Struktur des trockenen Rückstandes ist ersichtlich. Durch die direkte Einwirkung der Mikrowellen auf das Lösungsmittel trocknet der Rückstand von innen nach aussen und man erhält einen porösen trockenen Kuchen. Diese Trocknungsart erspart aufwendige Kontrollen, denn eine Sichtkontrolle von aussen genügt, um festzustellen, ob der $\mathrm{Ku}$ chen trocken ist. Diese trockenen Kuchen führt man der Verbrennung zu. Es wäre aber ideal, wenn sich eine Applikation finden liesse, bei welcher diese Kuchen zu einem Sekundärrohstoff aufgearbeitet werden könnten.

Das Beispiel in der Tabelle gilt für Destillationsrückstände aus einem Vakuumdestillator. Die Energiekosten für ein SOLIDEX-Gerät sind wie bereits erwähnt, sehr gering - bei $6000 \mathrm{Bh} / \mathrm{Jahr}$ betragen diese nur $c a$. Fr. 700.--/Jahr.

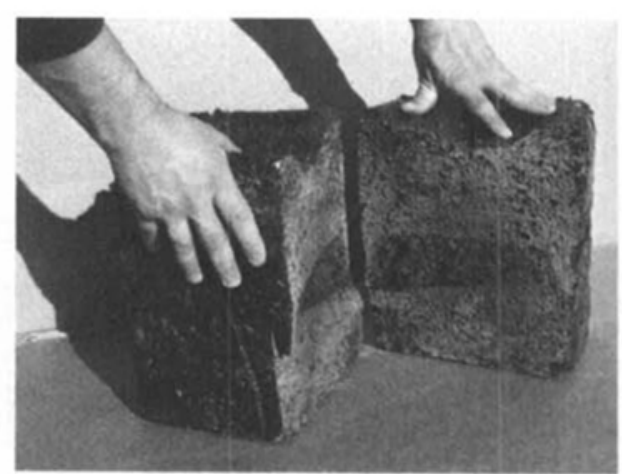

Fig. 7. Trockener Rückstand (Muster)

\section{Wirtschaftlichkeit}

Bei einem Anschaffungspreis eines SOLIDEX von $c a$. Fr. 80000 -- ergibt sich eine Amortisationszeit von weniger als einem Jahr. Ferner ist zu erwähnen, dass nicht nur die Vernichtungskosten, sondern ebenso die Handhabungskosten beträchtlich reduziert werden können, weil anstelle von aufwendigen Fasslagern das Aufstellen einer einfachen Mulde genügt, um die Destillationsrückstände zu lagern, bis diese der Entsorgung zugeführt werden.

\section{Schlussbetrachtung}

Die Rückgewinnung von Lösungsmitteln ist heute ein absolutes MUSS. Das SOLIDEX-Verfahren erlaubt es, nun CKW's auch dort wirtschaftlich zurückzugewinnen, wo konventionelle Verfahren bislang keine befriedigenden Resultate erbringen konnten oder gar gescheitert sind. Neben der Anwendung in der Leiterplattenfertigung sind selbstverständlich noch viele andere Applikationen für dieses Verfahren möglich.

\title{
Umdenken in der Abfallwirtschaft: Volkswirtschaftliche Aspekte des Materialrecycling
}

\author{
Gunter Stephan*
}

Veröffentlichungen zum Thema Abfall vermitteln nicht selten den Eindruck, wir stünden vor einem Müllnotstand. Diesen Eindruck kann ich nicht widerlegen oder verwischen. Dennoch scheint es mir wenig angebracht, in diesem Zusammenhang von einem Notstand zu sprechen. Denn das Wort Notstand suggeriert eine Situation,

\footnotetext{
* Korrespondenz: Prof. Dr. G. Stephan

Abteilung für Angewandte Mikroökonomie

Universität Bern

Gesellschaftsstrasse 27, CH-3012 Bern
}

in der der unmittelbare und massive Eingriff des Staates in die Wirtschaft unvermeidlich ist. Ich dagegen bin der Meinung, dass Abfallprobleme ökologisch und ökonomisch angemessener gelöst werden können, wenn der Staat nicht reglementierend in die Abfallwirtschaft eingreift, sondern Anreize schafft, die zur Vermeidung und Wiederverwertung von Abfällen führt.

Entwickelte Volkswirtschaften stehen heute vor zwei Problemen, deren Lösung sich gegenseitig behindert und scheinbar ausschliesst: Einerseits muss schonend mit der Natur und den knappen Deponieflächen umgegangen werden, um in der $\mathrm{Zu}$ kunft Leben und Wirtschaften zu ermöglichen. Andererseits soll die Innovationskraft der Wirtschaft gefördert werden, um Industriestandorte, Arbeitsplätze und den Wohlstand langfristig zu sichern. Beide Probleme können nur gelöst werden, wenn ein Umdenken in doppelter Hinsicht stattfindet: Erstens müssen bestehende Wirtschaftsstrukturen durch eine weniger roh- 